\title{
Propagation and dispersion of electrostatic waves in the ionospheric $E$ region
}

\author{
K. Iranpour, ${ }^{1}$ H. L. Pécseli ${ }^{1}$, J. Trulsen ${ }^{2}$, A. Bahnsen ${ }^{3 *}$, F. Primdahl ${ }^{3}$, K. Rinnert ${ }^{4}$ \\ 1 University of Oslo, Institute of Physics, P.O. Boks 1048 Blindern, N-0316 Oslo, Norway \\ 2 University of Oslo, Institute of Theoretical Astrophysics, P.O. Boks 1029 Blindern, N-0315 Oslo, Norway \\ 3 Technical University of Denmark, Department of Automation, DK-2800 Lyngby, Denmark \\ 4 Max-Planck-Insitut für Aeronomie, Postfach 20, D-37189 Katlenburg-Lindau, Germany
}

Received: 17 April 1996 / Revised: 6 November 1996 / Accepted: 10 December 1996

\begin{abstract}
Low-frequency electrostatic fluctuations in the ionospheric $\mathrm{E}$ region were detected by instruments on the ROSE rockets. The phase velocity and dispersion of plasma waves in the ionospheric $\mathrm{E}$ region are determined by band-pass filtering and cross-correlating data of the electric-field fluctuations detected by the probes on the ROSE F4 rocket. The results were confirmed by a different method of analysis of the same data. The results show that the waves propagate in the Hall-current direction with a velocity somewhat below the ion sound speed obtained for ionospheric conditions during the flight. It is also found that the waves are dispersive, with the longest wavelengths propagating with the lowest velocity.
\end{abstract}

\section{Introduction}

Low-frequency electrostatic waves in the auroral E region over northern Scandinavia were studied by instrumented rocket payloads during the ROSE campaign in 1988/1989. The experiment is discussed in a special issue of Journal of Atmospheric and Terrestrial Physics, Vol. 54 (1992). In the following, data from the ROSE F4 rocket in particular will be discussed. This rocket was launched approximately northwards in February 1989 from Kiruna, Sweden, while the electrojet $\boldsymbol{E} \times \boldsymbol{B} / B^{2}$ drift was approximately eastward. The basic data for the ionospheric conditions are summarized in Table 1. The ion temperature is assumed to be close to that of the neutral component. The neutral air temperature is measured by instruments on the rockets (Friker and Lübken, 1992). The value for the electron temperature is based on a model which is supported by EISCAT measurements (Kohl et al., 1992). Measurements on the rocket itself place an upper limit for the actual value of electron temperatures.
The waves observed in the collisional ionosphere at altitudes above $95 \mathrm{~km}$ were assumed to be excited by a two-stream type instability in a partially ionized gas, where DC electric fields are present, and modified by local gradients in plasma density. If the electric field strength exceeds a certain threshold, of the order of 20 $40 \mathrm{mV} / \mathrm{m}$, the electrojet current caused by this electric field gives rise to a linear instability for low-frequency electrostatic waves (Farley, 1963; Buneman, 1963). The standard expression for the dispersion relation in terms of frequency $\omega \equiv \omega_{r}+i \omega_{i}$ and wave vector $\boldsymbol{k}$ is

$$
\begin{aligned}
\omega_{r} & =\frac{k V_{d} \cos \alpha}{1+\varphi}, \\
\omega_{i} & =\frac{\varphi}{1+\varphi}\left[\frac{1}{v_{i}}\left(\omega_{r}^{2}-k^{2} C_{s}^{2}\right)+\frac{\omega_{r} \Omega_{e}}{k L_{n} v_{e}}\right],
\end{aligned}
$$

where

$\varphi=\frac{v_{e} v_{i}}{\Omega_{e} \Omega_{i}}\left(1+\frac{\Omega_{e}^{2} k_{\|}^{2}}{v_{e}^{2} k^{2}}\right)$,

with $v_{e, i}$ being the electron(ion)-neutral collision frequency, $\Omega_{e, i}$ the electron(ion) gyro-frequency, and where $\alpha$ is the angle between $\boldsymbol{k}$ and $\boldsymbol{V}_{d}$. The ion sound speed is $C_{s}$ and $\boldsymbol{V}_{d}=\boldsymbol{V}_{e}-\boldsymbol{V}_{i}$ is the difference between the electron- and ion-drift velocities to be derived from the $\boldsymbol{E} \times \boldsymbol{B}$ drift velocity modified by collisions with neutrals. At altitudes relevant to the present problem, the effect of ion-electron collisions is negligible compared to that of collisions with neutrals. A damping term due to recombination is ignored for simplicity. Equation (2) contains two contributions to the growth rate; the first parenthesis gives a two-stream type instability when $\omega_{r} / k>C_{s}$, while the second term accounts for a gradient driven instability, where $L_{n} \equiv n_{0}(x) / d_{x} n_{0}(x)$ is the scale length for the density variation in the $\boldsymbol{B}$ perpendicular direction. Note that $L_{n}$ has to be calculated with its sign included. Depending on the direction of $\nabla n_{0}$, a gradient in plasma density may have a stabilizing effect on the two-stream instability, but this 
Table 1. E-region and field parameters during the ROSE F4 flight

\begin{tabular}{|c|c|c|}
\hline Parameter & Value & Unit \\
\hline $\begin{array}{l}\text { Average Plasma density, } n_{0} \text {, } \\
\text { at an altitude of } 110 \mathrm{~km}\end{array}$ & $6.10^{10}$ & $\mathrm{~m}^{-3}$ \\
\hline Magnetic field, $B_{0}$ & 50 & $\mu \mathrm{T}$ \\
\hline Average DC electric field, $E_{0}$ & 45 & $\mathrm{mV} / \mathrm{m}$ \\
\hline Electron temperature, $T_{e}$ & 400 & $\mathrm{~K}$ \\
\hline Ion temperature, $T_{i}$ & 200 & $\mathrm{~K}$ \\
\hline Electron Debye-length, $\lambda_{D}$ & $6.10^{-3}$ & $\mathrm{~m}$ \\
\hline Average value of $E_{0} / B_{0}$ & 900 & $\mathrm{~m} / \mathrm{s}$ \\
\hline Vertical plasma density scale-length & 12.5 & $\mathrm{~km}$ \\
\hline Sound speed & 400 & $\mathrm{~m} / \mathrm{s}$ \\
\hline Electron cyclotron frequency, $\Omega_{e}$ & $9.3 \cdot 10^{6}$ & $\mathrm{rad} / \mathrm{s}$ \\
\hline $\begin{array}{l}\text { Ion cyclotron frequency, } \Omega_{i} \text {, corresponding } \\
\text { to an average mass of } 31 \mathrm{amu}\end{array}$ & 180 & $\mathrm{rad} / \mathrm{s}$ \\
\hline $\begin{array}{l}\text { Ion-neutral collision frequency, } v_{i n} \text {, at } \\
\text { an altitude of } 110 \mathrm{~km}\end{array}$ & 600 & $\mathrm{~s}^{-1}$ \\
\hline $\begin{array}{l}\text { Electron-neutral collision frequency, } v_{e n} \text {, at } \\
\text { an altitude of } 110 \mathrm{~km}\end{array}$ & $1.5 \cdot 10^{4}$ & $\mathrm{~s}^{-1}$ \\
\hline
\end{tabular}

effect will usually be restricted to the long wavelength part of the spectrum. The gradient contribution to the growth rate in Eq. (2) should be considered with some care, because of the assumed constancy of $L_{n}$. In realistic situations the exponential approximation to the density gradient may not be appropriate. The exponential approximation will often break down for long wavelengths, which is where the gradient term in Eq. (2) is most important. Equations (1) and (2) implicitly assume $k_{\|}^{2} \ll k_{\perp}^{2}$ and also that the growth rate $\omega_{i}$ is small. Consequently, Eqs. (1) and (2) are valid only near threshold. Kudeki et al. (1982) give results without this restriction on the growth rates, but on the other hand they ignore ion inertial effects. With $k_{0} \equiv\left(v_{i} / \Omega_{i} L_{n}\right)$ $/(1+\varphi)$ they give for waves propagating in the direction normal to $\boldsymbol{B}$ a dispersion relation in the form

$\omega_{r}=\frac{k^{3} V_{d}}{(1+\varphi)\left(k^{2}+k_{0}^{2}\right)}$

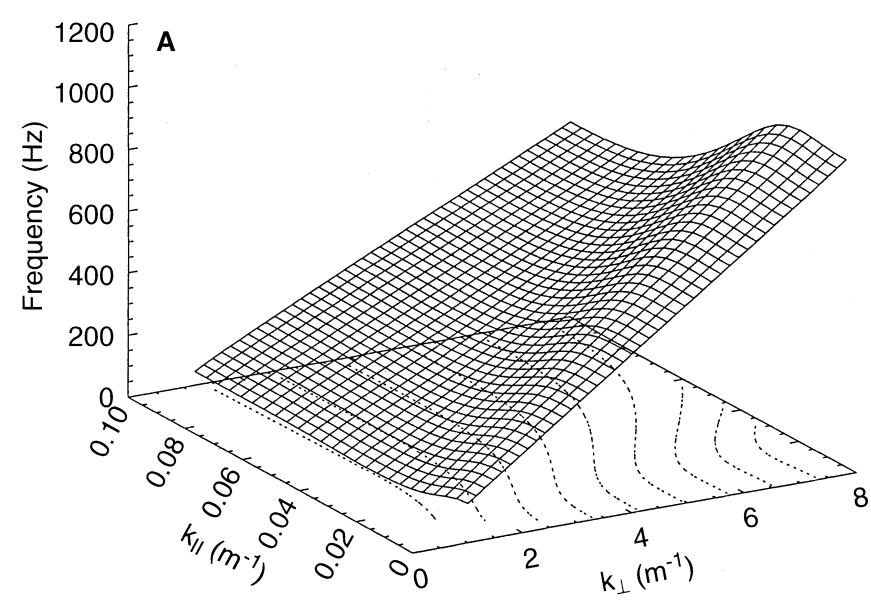

Fig. 1A, B. Numerically obtained dispersion relation for the FarleyBuneman instability. A The real part of the frequency; $\mathbf{B}$ the imaginary part, as functions of wave vector $\boldsymbol{k}$ with components $k_{\|}$and $k_{\perp}$ along $\boldsymbol{B}$ and $\boldsymbol{E} \times \boldsymbol{B}$, respectively. Parameters are $6.10^{10} \mathrm{~m}^{-3}$ for density, 450 and

$$
\omega_{i}=\frac{k_{0} k^{2} V_{d}}{(1+\varphi)\left(k^{2}+k_{0}^{2}\right)} .
$$

These results are based on the analysis presented by Sudan et al. (1973), but also Rogister (1972) presented similar expressions. Evidently, within the approximations in Eqs. (3) and (4), the two-stream or FarleyBuneman contribution to the instability no longer appears. Note that the waves are dispersive, with longest wavelengths having the smallest phase velocity, according to Eq. (3). The growth rate increases with wave number according to Eq. (4). A damping due to recombination can be introduced ad hoc by subtracting $2 \alpha n_{0}$ from Eq. (4) or (2), with $\alpha \approx 3 \cdot 10^{-3} \mathrm{~m}^{3} / \mathrm{s}$ being the dissociative recombination coefficient (Kudeki et al., 1982).

Since the measured DC electric fields are significantly above the threshold value for the two-stream type instability, we performed a detailed stability analysis for these particular waves, extending the analysis used in obtaining Eqs. (1) and (2) by relaxing the restriction of small growth rates implied in the derivation of these relations. The gradient contribution is ignored here since we have no a priori information about density gradients in the direction perpendicular to the magnetic field. As a first attempt at an interpretation we want to find out to what extent the observations can be explained by the Farley-Buneman instability alone. In Fig. 1 we show a numerically obtained dispersion relation, with the real and the imaginary parts of the frequency shown as functions of wave vector in the plane determined by the magnetic field $\boldsymbol{B}$ and the $\boldsymbol{E} \times \boldsymbol{B}$ direction. The growth rates can be rather large and the assumptions inherent in Eqs. (1) and (2) are violated for the relevant electrojet conditions. The basic equations for the analysis underlying Fig. 1 are the linearized continuity, momentum, and temperature equations for electrons and ions, with the dynamics coupled through Poisson's equation.

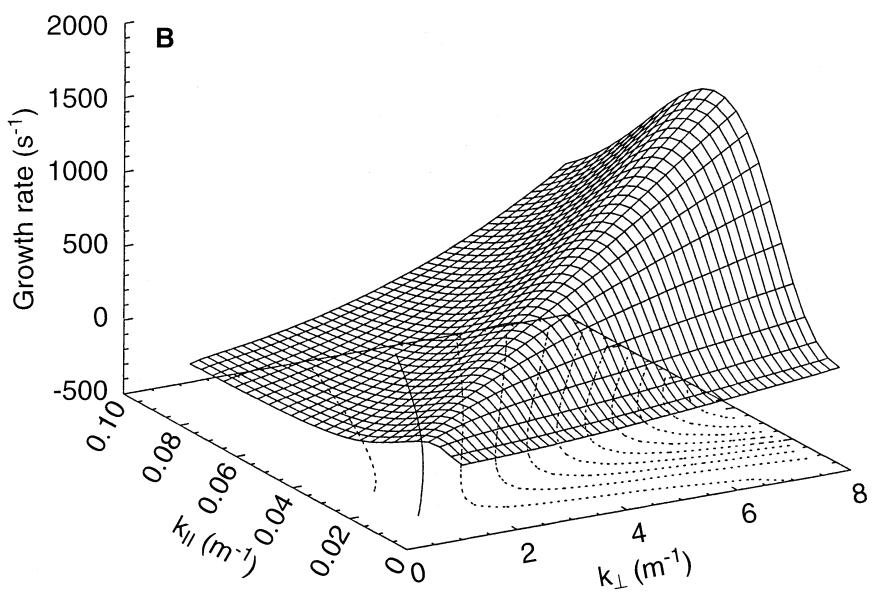

and $250 \mathrm{~K}$ for electron and ion temperatures, respectively, $\boldsymbol{B}_{0}=50 \mu \mathrm{T}, E_{0}=40 \mathrm{mV} / \mathrm{m}, v_{i}=600 \mathrm{~s}^{-1}, v_{e}=1.5 \cdot 10^{4} \mathrm{~s}^{-1}$ appropriate for an altitude of $110 \mathrm{~km}$. We used an effective ion mass corresponding to $31 \mathrm{amu}$ as obtained from standard tables 
Details of the analysis need not be given here, we only note that some fine structures in the dispersion relation can be explained by a transition from adiabatic to isothermal electron dynamics as the wave vector turns away from the $\boldsymbol{B}$-perpendicular direction (Pécseli et al., 1989). Ion viscosity and finite ion Larmor radius effects are ignored. The real part of the numerically obtained dispersion relation is in overall agreement with the simple results of Eq. (1), while the growth rates of Eq. (2) are somewhat modified. In particular, it is observed that the linearly most unstable waves propagate at a small angle to the $\boldsymbol{E} \times \boldsymbol{B} / B^{2}$ velocity. This is due to the transition from adiabatic to isothermal electron dynamics, as mentioned before. The particular features included in our analysis will be especially important near the threshold. Detailed studies of these electrojet instabilities are of general interest because these waves provide an example where a large-scale electric field directly excites small-scale fluctuations, without an intermediate energy cascade. Equations (1)-(4) and the results summarized in Fig. 1 will be used as a guide for discussing the experimental results.

The purpose of the present study is to analyze parts of the data obtained by rocket-borne sensors launched into the $\mathrm{E}$ region. Characteristic phase velocities and wavelengths of these waves are estimated. The data are nonstationary due to the high speed of the rocket through a plasma with temporal and spatial inhomogeneities. Consequently, the analysis is performed on short time-sequences with care taken to ensure the statistical significance of the results. Particular attention to this question was given by Pécseli et al. (1993) in their analysis of $\mathrm{E}$ region data, and elaborated in more detail by Pécseli and Trulsen (1993) in a similar context. The ELF signals discussed in the following were first analyzed by Rinnert (1992).

\section{Electric Field measurement}

The three components of the AC electric field in the frequency range $120 \mathrm{~Hz}-3.5 \mathrm{kHz}$ were detected by segmented spherical probes (KUSO). Electron-density fluctuations were detected by two retarding potential analyzers, one pointing along the rocket axis and one in the perpendicular direction. The ELF signals in the frequency range $0-600 \mathrm{~Hz}$, to be analyzed in the following, were obtained by means of gold-plated spherical probes of $5 \mathrm{~cm}$ diameter, mounted on two pairs of booms, one near the top of the payload (labelled 1 and 2) and the others $185 \mathrm{~cm}$ lower (labelled 3 and 4), oriented at an angle of $90^{\circ}$ with respect to the first pair, see Fig. 2 and also the description by Rose et al. (1992). The total length of each pair of booms was $360 \mathrm{~cm}$. In the following only data from the F4 rocket will be analyzed; it had an altitude of $123 \mathrm{~km}$ at apogee. Its horizontal velocity component was approximately 200 $\mathrm{m} / \mathrm{s}$. The spin period was approximately $0.6 \mathrm{~s}$ and the coning half angle approximately $2^{\circ}$ with a coning period of approximately $5.8 \mathrm{~s}$. These quantities vary only slowly with time, so they can be considered as essentially

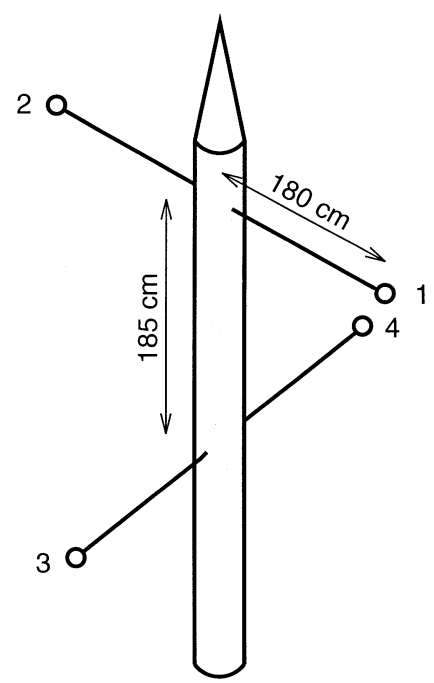

Fig. 2. Probe configuration of a rocket from the ROSE campaign. A more detailed figure describing the rocket is given by Rose et al. (1992)

constant during the time-interval that the rocket spent in the ionospheric E region.

The electric fields were obtained by the rocket-borne sensors designed to measure both DC and low-frequency AC electric fields. The sampling rate is $4 \mathrm{kHz}$, but the data file is reduced to one sample for every $0.5 \mathrm{~ms}$ (2000 samples/s). DC electric field values in the range 30-70 $\mathrm{mV} / \mathrm{m}$ were reported for the F4 flight. At an upleg altitude of $110 \mathrm{~km}$ the observed value was $45 \mathrm{mV} / \mathrm{m}$ on average, for the downleg part at the same altitude it was $52 \mathrm{mV} / \mathrm{m}$. These results are discussed by Rinnert (1992) and compared with results from the STARE and EISCAT radars obtained for somewhat higher altitudes, see Fig. 3. A source of uncertainty for the DC electric field measurements is the variation in the local field introduced by the rocket itself. These have been discussed by for instance Rohde et al. (1993), but seem to be of importance only at the sheaths in vicinity of the body, not at the position of the boom-mounted probes.

Fluctuating electric fields with RMS levels in excess of $5 \mathrm{mV} / \mathrm{m}$ were also detected in situ by the four probes (Rinnert, 1992). If each probe has a potential $\phi_{j}$, the permutation of all the possible potential differences $\Delta \phi$ between these four probes yields six combinations. For ideal conditions there would be a redundancy in these six signals. For later reference, the six combinations are denoted $\phi_{1}-\phi_{2}=U_{6}, \phi_{4}-\phi_{3}=U_{5}, \phi_{1}-\phi_{4}=U_{4}$, $\phi_{2}-\phi_{3}=U_{3}, \phi_{1}-\phi_{3}=U_{2}, \phi_{2}-\phi_{4}=U_{1}$. Later, the direction of the wave fronts will be given with reference to the booms connecting probe pairs. In Fig. 4 we show the relative variation of the RMS power of the potential fluctuations $U_{5}(t)$ as a function of time after launch as well as altitude. The data were numerically high-pass filtered at $5 \mathrm{~Hz}$ to remove the spin frequency and its lowest harmonics. Successive time-sequences of $64 \mathrm{~ms}$ duration were analyzed to give the local RMS values. Due to rocket spin, Fig. 4 is representative also for the signal $U_{6}(t)$. Any remaining modulational effect from the rocket spin can not be discerned in Fig. 4. We note 


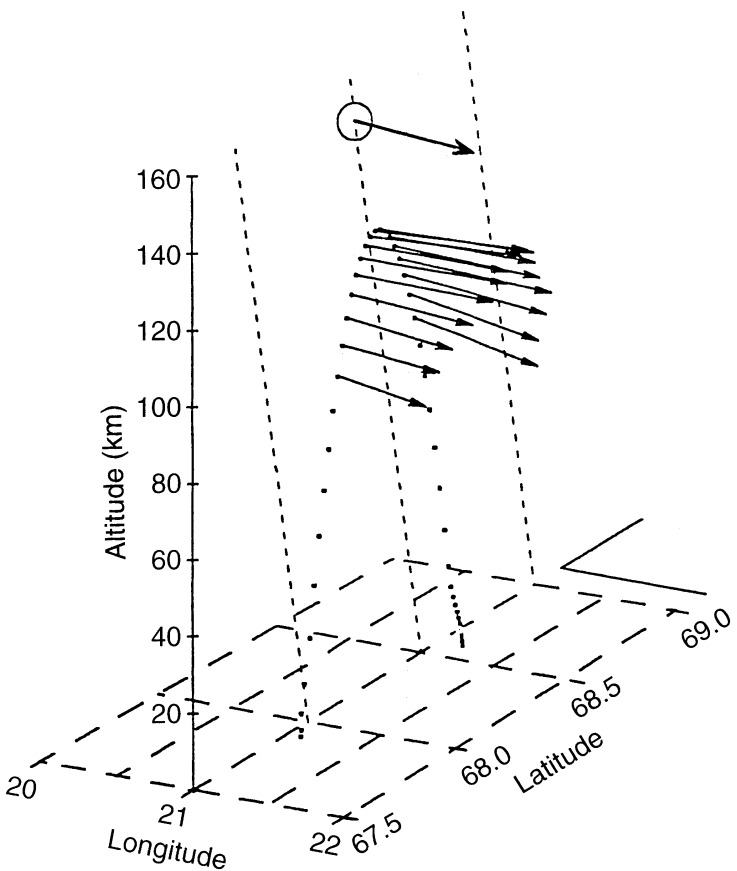

Fig. 3. Diagram of local drift vectors obtained from the ROSE F4 rocket data assuming a pure $\boldsymbol{E} \times \boldsymbol{B}$ drift. The arrow with a circle gives the direction of the plasma drift as detected by EISCAT at the indicated altitude. The length of that arrow corresponds to a deduced DC electric field of $45 \mathrm{mV} / \mathrm{m}$, for more details see the analysis of Rinnert (1992). Dashed lines indicate the magnetic field, dots the rocket trajectory. The magnetic field dip-angle is $77^{\circ}$

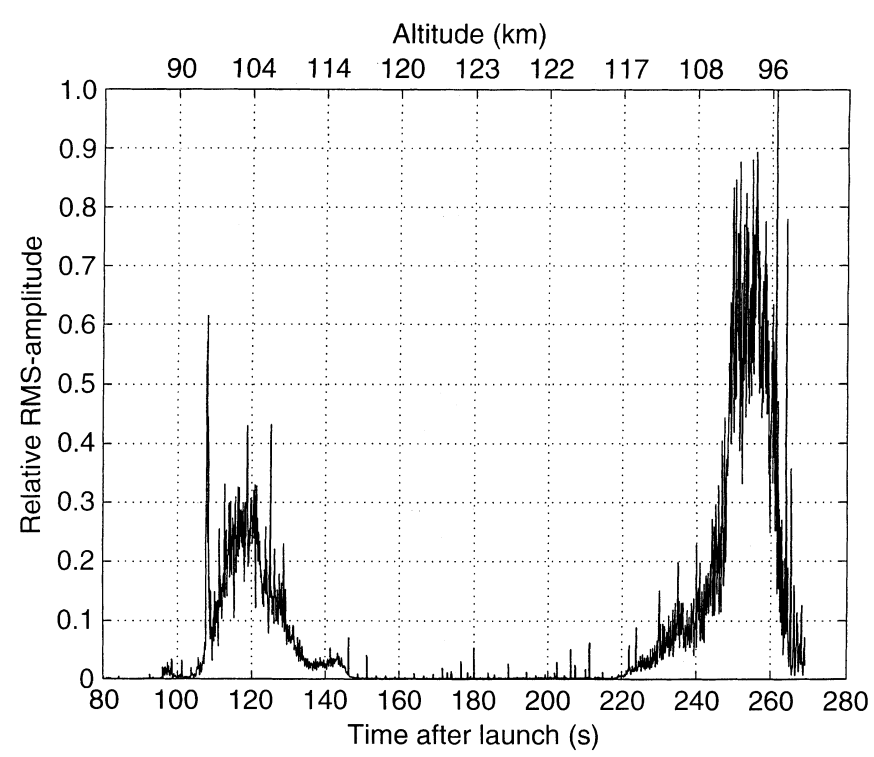

Fig. 4. Root-mean-square power of the fluctuations in potential $U_{5}$ in arbitrary units as a function of time after launch. In the top of the figure the corresponding altitudes are given

significant wave activity which is clearly confined to the ionospheric E region. It is noted that the RMS power takes its largest value at a downleg position where the largest DC electric fields are observed. In Fig. 5 we show the spectral characteristics of fluctuations in density as well as electric fields for upleg and downleg conditions.
The observed fluctuations in electric field are accompanied by corresponding density fluctuations, as expected for low-frequency, acoustic-like waves.

Interpreting the spectra from the different $U_{j}(t)$ signals, we note that these give only approximations to the component of the electric field in the direction defined by each probe pair. As a first approximation, consider the signal as a superposition of independent plane waves, all propagating in the same direction

$$
\begin{aligned}
\boldsymbol{E} \equiv & -\nabla \phi(\mathbf{r}, t) \\
= & -\nabla \sum_{j}\left(a_{j} \cos \left(\omega_{j} t-\mathbf{k}_{j} \cdot \mathbf{r}\right)\right. \\
& \left.+b_{j} \sin \left(\omega_{j} t-\mathbf{k}_{j} \cdot \mathbf{r}\right)\right) .
\end{aligned}
$$

Assuming for the sake of argument that the probe pair is directed along the $x$-axis, we obtain for instance

$$
\begin{aligned}
U_{5}(t)= & \sum_{j}\left(a_{j}\left[\cos \left(\omega_{j} t-k_{j},{ }_{x} x_{3}\right)-\cos \left(\omega_{j} t-k_{j},{ }_{x} x_{4}\right)\right]\right. \\
& \left.+b_{j}\left[\sin \left(\omega_{j} t-k_{j},{ }_{x} x_{3}\right)-\sin \left(\omega_{j} t-k_{j},{ }_{x} x_{4}\right)\right]\right) .
\end{aligned}
$$

With $\Delta=x_{4}-x_{3}$ being the projection of the probe separation vector on the normal to the phase fronts and $R=\frac{1}{2}\left(x_{4}+x_{3}\right)$, we find

$$
\begin{aligned}
-\frac{1}{\Delta} U_{5}(t)= & \frac{2}{\Delta} \sum_{j}\left(a_{j} \sin \left(\omega_{j} t-k_{j, x} R\right)\right. \\
& \left.-b_{j} \cos \left(\omega_{j} t-k_{j, x} R\right)\right) \sin \left(\frac{1}{2} k_{j, x} \Delta\right) \\
= & \sum_{j} E_{x, j}(t, R) \frac{\sin \left(\frac{1}{2} k_{j, x} \Delta\right)}{\frac{1}{2} k_{j, x} \Delta} .
\end{aligned}
$$

Consequently, for the simple model of Eq. (5) the probe signal can be interpreted as the electric field multiplied with a filter $\sin \left(\frac{1}{2} k_{x} \Delta\right) /\left(\frac{1}{2} k_{x} \Delta\right)$, as discussed by Kelley and Mozer (1973). The power spectrum of the probe signal can be understood as the electric field power spectrum multiplied by

$$
\left(\frac{\sin \left(\frac{1}{2} k_{x} \Delta\right)}{\frac{1}{2} k_{x} \Delta}\right)^{2} \text {. }
$$

For the case of a "white" spectrum for the electric field, the envelope of the signal power spectra will therefore appear as $k_{x}^{-2}$ for short wavelengths $\left|k_{x} \Delta\right| \gg 1$, or $\omega^{-2}$ for large frequencies, provided we can assume that frequencies and wave numbers are proportional. Only when $\left|k_{x} \Delta\right| \ll 1$ do we have a one-to-one correspondence between the potential difference between the two probes and an electric-field component in the plasma. Neglecting coning effects, the rocket spin gives a modulation $\Delta=\Delta_{0} \cos \Omega t$ for the projection of the probe separation on a reference direction perpendicular to the rocket axis. Consequently, the signal envelope spectral index at a given frequency may vary with the rocket spin phase. At times when the direction of the probe pair in question is close to being parallel to the phase fronts, the entire spectrum becomes poorly defined. At the expense of a clumsy notation, the 

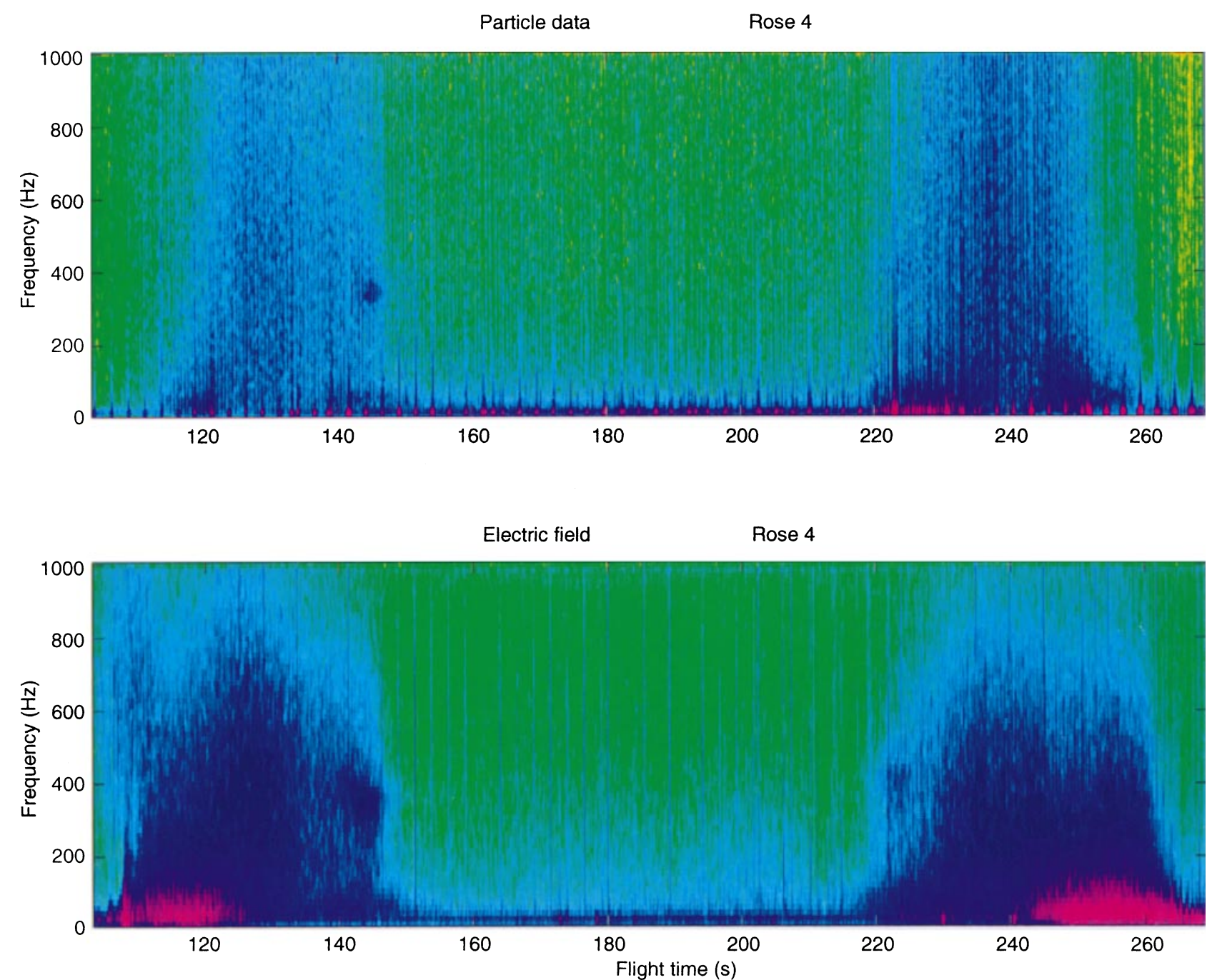

Fig. 5. Spectral distributions of the electroject fluctuations as a function of time after launch. See Fig. 4 for the corresponding altitudes. The top frame shows fluctuations in plasma density ("particle data") obtained by the current to a retarding potential analyzer on the rocket (Rose et al., 1992). The lower frame is obtained

foregoing arguments can readily be generalized to more complicated wave models, e.g. one where the waves propagate in a directional cone. The results already outlined will suffice for the present purpose.

\section{Experimentally obtained dispersion relation}

Some of the basic characteristics for describing the observed electric field fluctuations are their speed and direction of propagation as a function of frequency. Generally, the waves are described by dispersion relations, obtained analytically on the basis of linearized equations as discussed in the Introduction. These analytical results predict that the waves are essentially acoustic and that the real part of the dispersion relation can be approximated by $\omega=V \boldsymbol{k} \cdot \hat{\boldsymbol{e}}$, where $\hat{\boldsymbol{e}}$ is in the $\boldsymbol{E} \times \boldsymbol{B}$ direction and $V$ is a constant phase velocity slightly below the $\boldsymbol{E} \times \boldsymbol{B} / B^{2}$ velocity. It will be demon- by the signal from one probe pair as in Fig. 4. The fluctuations at a frequency close to $400 \mathrm{~Hz}$ occur at a well-localized altitude, and fall outside the scope of the present study. Power spectral intensities are in arbitrary units

strated in the following that the ionospheric data from the ROSE F4 rocket are in some respects in contradiction with such a linear dispersion relation, even though the DC electric field in the ionosphere is well above the threshold value of the two-stream instability, as evidenced by the numerical results in Fig. 1.

\subsection{Results from cross-correlation}

Figure 6 shows the normalized cross-correlation between the $U_{1}$ and $U_{2}$ signals over an interval of the flight time in the form of a contour map. The frequency range is $5-1000 \mathrm{~Hz}$. The sinusoidal variation of the time-delay, clearly seen in Fig. 6, closely follows the spin phase of the rocket. This can be taken as evidence for a unidirectional propagation, as demonstrated by simple geometrical arguments (Bahnsen et al., 1978; Pécseli et al., 1989, 1993). In case the waves were to propagate 


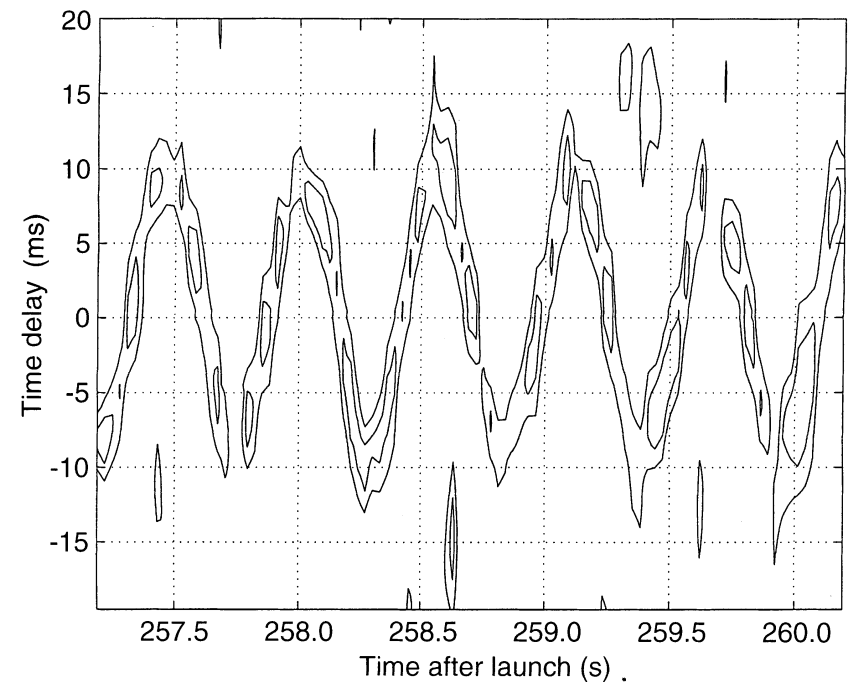

Fig. 6. Contour plot of normalized cross-correlation of the $U_{1}$ and $U_{2}$ potential differences is shown over an interval of ROSE F4 downleg flight time. See Fig. 4 for the corresponding altitudes. The frequency range is $5-1000 \mathrm{~Hz}$. The distance between the $U_{1}$ and $U_{2}$ measuring probe sets is approximately $2.54 \mathrm{~m}$. For simplicity, only the 0.5 and 0.75 contours are shown

in a wide cone of directions, a smearing out of the crosscorrelation would result.

The time-delay obtained from this cross-correlation can be used to determine a component of the apparent speed of propagation in the rocket frame of reference since the distance between the two probe pairs is known a priori. From the probe pairs $(2,4)$ and $(1,3)$ we find the velocity component perpendicular to the rocket axis. For a well-defined direction of propagation, the maximum value of the time-delay of the cross-correlation will occur at the spin phase when a given probe pair is parallel to the characteristic direction of wave propagation. An apparent velocity component calculated in this way (Iranpour et al., 1993), varies slowly with altitude in the range $175-250 \mathrm{~m} / \mathrm{s}$, with an abrupt increase to approximately $300-350 \mathrm{~m} / \mathrm{s}$ around $105 \mathrm{~km}$ altitude at upleg conditions, see results in Fig. 7.

We also analyzed the cross-correlation of the signals $U_{5}$ and $U_{6}$ to obtain an estimate for the component of the apparent velocity $v_{\|}$along the rocket axis, see results in Fig. 8. The positive direction is taken antiparallel to the rocket velocity. We found $v_{\|}$to be in the range $1 \cdot 10^{3}-4 \cdot 10^{3} \mathrm{~m} / \mathrm{s}$, i.e. much larger than the transverse velocity $v_{\perp}$ previously discussed. The rocket velocity is $V_{0} \approx 10^{3} \mathrm{~m} / \mathrm{s}$. The uncertainty in the estimate for the parallel velocity $v_{\|}$in the rocket frame arises because of the small time-delays involved. Within this uncertainty we may argue that the direction of propagation of the waves is perpendicular to the rocket axis within an angle of $\pm 10-20^{\circ}$. The absolute value of the phase velocity $V_{p h}$ can, for one wave number component of a plane wave, be obtained as $V_{p h}=\boldsymbol{k} \cdot \boldsymbol{V}_{0} / k+v_{\perp} v_{\|} / \sqrt{v_{\perp}^{2}+v_{\|}^{2}}$. We used $\tan \theta=v_{\|} / v_{\perp}$, with $\theta$ being the angle of propagation direction with respect to the rocket axis. With $v_{\|} \gg v_{\perp}$, i.e. $\theta \approx 90^{\circ}$, we have for the present experiment that $V_{p h} \approx v_{\perp}$. For propagation strictly

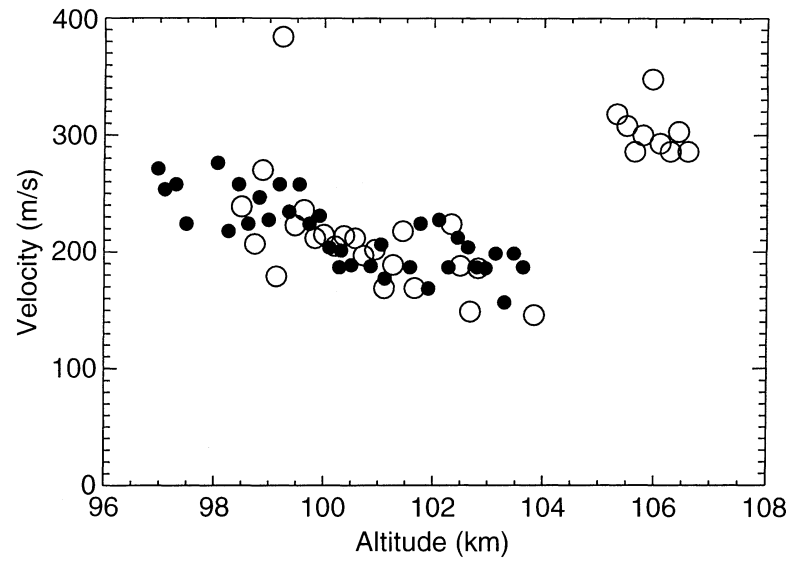

Fig. 7. Propagation velocity component in the direction perpendicular to the rocket axis obtained from the maximum time-delay of cross-correlation of the signals $U_{1}$ and $U_{2}$, see also Fig. 6 . The results are shown as a function of altitude. Open circles are for upleg conditions, filled circles for downleg. A possible uncertainty for the interpretation arises from vibrations in the booms of the detecting probes. As the RMS-level of the fluctuations varies gradually with altitude we may exclude wave nonlinearities as the cause of the abrupt change in phase velocity observed at upleg conditions

perpendicular to the rocket axis we have $v_{\|}$infinite. Since the waves can be assumed to propagate in the direction perpendicular to the rocket axis we thus argue that the Doppler correction is negligible and that the observed velocities are also close to those in the rest frame. Recall that the coning angle of the rocket is negligible in this context. The observed phase velocity is significantly below the $\boldsymbol{E} \times \boldsymbol{B} / B^{2}$ drift velocity and closer to the ion acoustic speed, which for the relevant plasma conditions is approximately $450 \mathrm{~m} / \mathrm{s}$. An uncertainty in

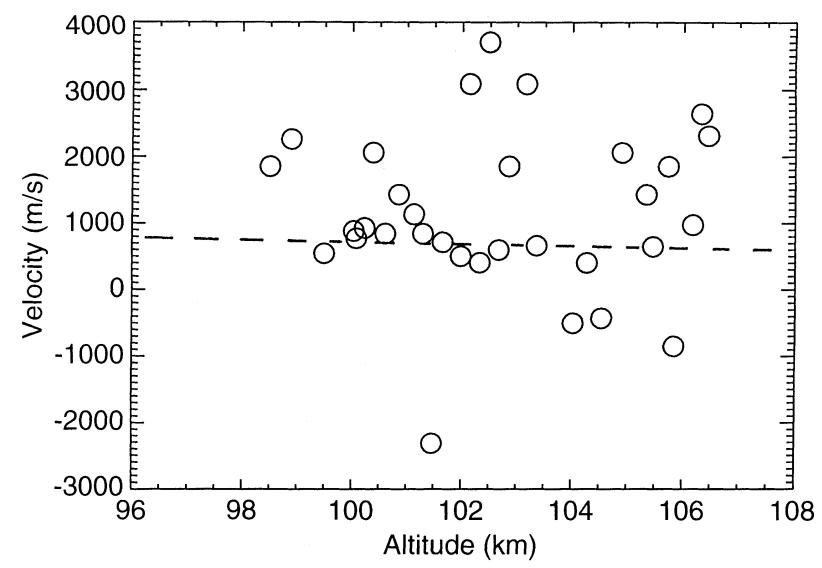

Fig. 8. Propagation velocity component in the direction along the rocket axis obtained from the maximum time-delay of crosscorrelation of the signals $U_{5}$ and $U_{6}$, see also Figs. 6 and 7. The results are shown as a function of altitude for upleg conditions. The dashed line shows the slowly varying vertical component of the rocket velocity. Points clustering around this velocity may indicate the presence of structures in the plasma density, which, in the rocket frame, will appear as propagating backwards with the rocket speed. Also points with forward propagation velocity can be expected, due to detection uncertainty when we observe essentially plane structures propagating in a direction perpendicular to the rocket 
this number originates mainly from the uncertainty in the electron temperature.

Referring to the standard theoretical results concerning the real part of the dispersion relation for the FarleyBuneman instability, see also Fig. 1, a characteristic velocity slightly below the $\boldsymbol{E} \times \boldsymbol{B} / B^{2}$ velocity is expected. Experimentally, the waves are often seen to propagate with a characteristic velocity close to the ion-acoustic sound speed (Pécseli et al., 1989). The bulk velocities observed here are even lower, in particular at altitudes below $105 \mathrm{~km}$, as already mentioned. Bahnsen et al. (1978) observed similar low phase velocities in their studies of rocket data obtained in the ionospheric $\mathrm{E}$ region over Greenland. Wave propagation velocities were also investigated by the ERRIS campaign in 1988 and 1989 (Pfaff et al., 1992) for conditions similar to the ROSE campaign. Rocket frame velocities around $900 \mathrm{~m} / \mathrm{s}$ were reported, with narrow spectral features corresponding to wavelengths of approximately $2.4 \mathrm{~m}$ as derived from an analysis of the downleg data of the first ERRIS rocket. For this case the DC electric field was in excess of $100 \mathrm{mV} / \mathrm{m}$, i.e., noticeably larger than for the conditions of the ROSE F4 rocket. LaBelle et al. (1986) obtained results by cross-correlating signals from two spatially separated fixed-bias density probes, and obtained propagation velocities in the range of $400-600 \mathrm{~m} / \mathrm{s}$. In their case, the DC electric field was approximately $25 \mathrm{mV} / \mathrm{m}$, so there is little distinction between the sound speed and their $\boldsymbol{E} \times \boldsymbol{B} / B^{2}$ velocity.

\subsection{Band-pass filtered correlations}

Rather than estimating a phase velocity $V_{p h}$ for each frequency component, the use of the cross-correlation function as described only gives a one-point estimate for the entire dispersion relation. This use of the crosscorrelation evidently emphasizes the wave component with the largest amplitude. To obtain the full dispersion relation, extending over a wide frequency range, the data were filtered sequentially to frequency bands of width $\Delta f$ when calculating $V_{p h}=V_{p h}(f)$. Phase velocities thus obtained refer to the particular frequency band. In this way the full dispersion relation can be determined. When selecting the bandwidth we made sure that $\Delta f>1 / T$, with $T$ being the duration of the signal sequences. The filtering is carried out numerically. An example of $V_{p h}$ as a function of $f$ obtained by this method as applied to a time-interval of ROSE F4 electric-field data, is shown in Fig. 9. Full and dashed lines refer to up- and downleg conditions. These two regions are horizontally separated by approximately $25 \mathrm{~km}$. Due to the uncertainty caused by a neutral wind, the phase velocity may be different in the rest frame of the neutral component. It is possible that neutral winds present give rise to ion drifts of sufficient magnitude to explain the observed reduction of phase velocities compared to the estimated ion sound speed. The required wind speeds are of the order of $100 \mathrm{~m} / \mathrm{s}$, which are large, but not unreasonable (Rees and FullerRowell, 1988). It is unlikely, however, that the observed

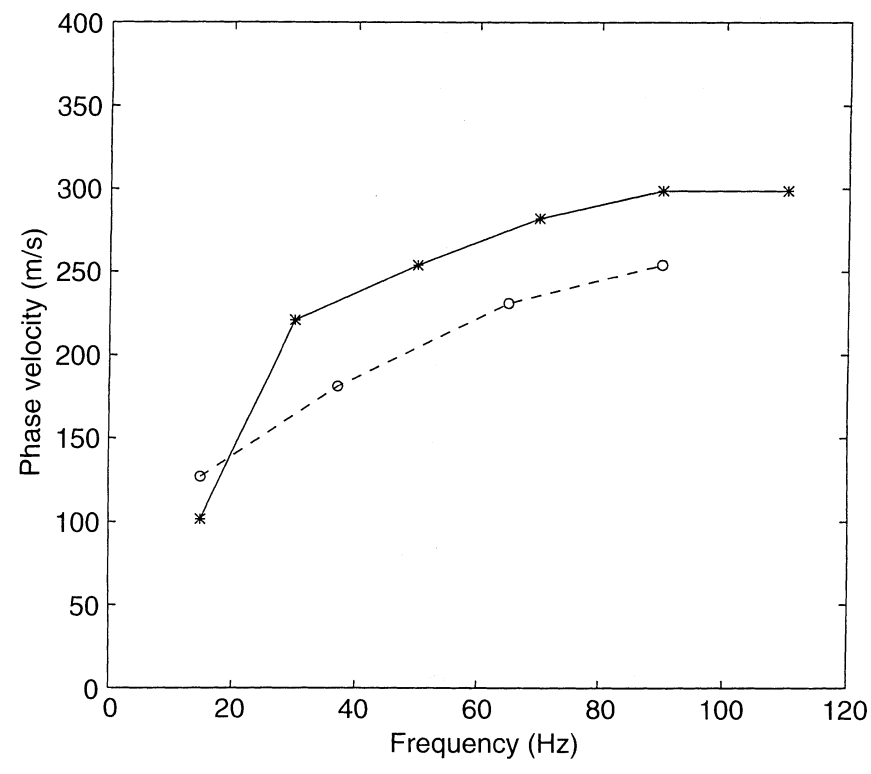

Fig. 9. Dispersion relation, i.e., phase velocity for various frequencies, obtained by band-pass filtering the data prior to the calculation of the cross-correlation. Solid line refers to upleg conditions, dashed line to downleg. The data are obtained for an altitude interval of approximately $2 \mathrm{~km}$, centered around $102 \mathrm{~km}$ altitude

wave dispersion is caused by a neutral wind, since this would give the same change in phase velocity at all frequencies.

\subsection{Cross-phase spectrum}

To provide further evidence for the observed wave dispersion, we also analyzed the cross-phase spectrum. The Fourier transform of the cross-correlation may be written in the form $S_{12}(\omega) \exp (i \Theta(\omega))$, where $S_{12}(\omega)$ is the cross-power spectrum and $\Theta(\omega)$ is the cross-phase spectrum. In the case of frozen turbulence, where structures in the plasma are convected without change in shape, $S_{12}(\omega)$ would be identical to the power spectrum of the fluctuations and $\Theta(\omega)=\omega \tau$, with $\tau$ being the time-delay for the signal propagating from the first to the second detector. In general, the cross-phase can be interpreted as the phase-delay of individual frequency components, and thus effectively representing the dispersion relation for the essentially one-dimensional situation observed, recalling again the experimental evidence for an almost unidirectional propagation of the fluctuations.

The projection $\Delta_{0} \sin \theta$ of the separation of two probe pairs on the normal to the wave front, changes with the rotation of the rocket, where $\theta=\theta(t)$ is the angle between the normal to the wave front and the probe-pair axis. It is clear that when $\theta$ approaches $\pm \pi / 2$, each probe pair lies on the same wave front. The probes then ideally measure the same potential and the corresponding potential difference would be negligible. In case we have a monochromatic wave hidden in an additive Gaussian noise, the probes should measure only the Gaussian background noise for $\theta \sim \pm \pi / 2$. On the other 
hand, when $\theta$ approaches zero, the direction determined by the two probes in a pair is approximately along the direction of the wave propagation, and both pairs ideally measure the maximum electric field. In this case $\Delta$ approaches zero and the velocity estimate is undefined. This means that the optimum angle $\theta$ between the axes and the normal to the wave front for determining a wave number or phase velocity is near the middle of the range $[0, \pm \pi / 2]$. The estimate of the relationship between the phase velocity and frequency, shown in Fig. 10, is based on a few averages centered around this optimum range. We also find that this method for obtaining the dispersion relation gives clear indications for the lower frequencies having the smallest phase velocities, in agreement with Fig. 9. The cross-power spectrum confirms the observation that the smallest frequencies have the smaller amplitudes. The phase velocity deduced from the cross-phase corresponding to frequencies with the largest cross-power is close to the bulk velocity obtained from cross-correlations as described in connection with Fig. 6. This is expected, since that method emphasizes the largest amplitudes. Since the cross-phase is obtained as the ratio of the imaginary and real parts of the spectrum, it becomes uncertain for frequencies where the cross-power is small. The dispersion relations shown in Figs. 9 and 10 do not agree in all details, but considering the differences in the methods of analysis and the statistical uncertainties involved, we find the overall agreement convincing. Assuming that the up- and downleg curves refer to essentially similar plasma conditions, we argue that the differences between the curves (full and dashed lines) in Figs. 9 and 10 can be taken as a measure of the uncertainty in the estimate of wave dispersion.

The cross-phase spectrum was also obtained by Pécseli et al. (1989). In their data, they found evidence for weak or negligible wave dispersion. In that case,

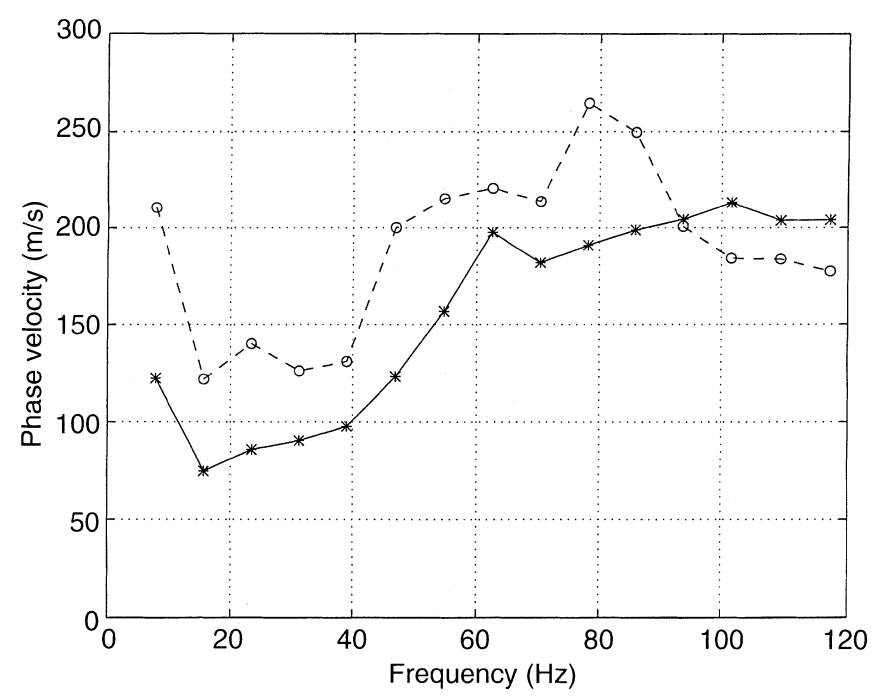

Fig. 10. The phase velocity as a function of frequency obtained from the cross-phase spectrum. Solid line refers to upleg conditions, dashed line to downleg. The data are obtained for an altitude interval of approximately $2 \mathrm{~km}$, centered around $102 \mathrm{~km}$ altitude however, the waves were propagating at a speed close to, and in some cases even in excess of, the ion sound speed for the appropriate ionospheric temperatures. It might be anticipated that there were some differences in the ionospheric conditions in the two cases, where one possible candidate might be localized gradients in the plasma density in the direction perpendicular to the magnetic field during the ROSE F4 flight.

The observed dispersion of waves propagating subsonically in a well-defined direction is not easily accommodated within a simple theory for a saturated twostream instability. An enhanced, anomalous collision frequency can give rise to a reduced phase velocity, which can be close to the ion acoustic speed (Primdahl, 1986; Primdahl and Bahnsen, 1985). It is interesting that laboratory experiments (John and Saxena, 1975; Mikkelsen and Pécseli, 1980; Pécseli et al., 1983) have shown dispersive properties of waves under conditions similar to those observed in the electrojet in the present study.

\subsection{Direction of propagation}

We also analyzed the variations in the direction of propagation of the fluctuations. From the cross-correlations like those shown in Fig. 6, we can determine the spin and coning phase of the rocket at the time when the probe pairs in question are aligned in the direction giving maximum time-delay. The change in phase of the rocket between successive alignments is calculated. Phases for up- and downleg conditions obtained by this method are shown in Fig. 11. Ideally, if the waves propagated in exactly the same direction and the method of analysis was without uncertainty, all points should be lying on a horizontal line. Instead we note a nontrivial scatter, largest for the upleg conditions. We conclude that the waves are seemingly unidirectional within $\pm 25^{\circ}$ for upleg and $\pm 15^{\circ}$ for downleg conditions. The figures were obtained by determining the rocket spin-phase at every half-turn, i.e., at alternating positive and negative peak delays in the cross-correlation. If only the phases for a full turn are used, the scatter in points for the upleg conditions is noticeably reduced, and will correspond approximately to the scatter at downleg conditions. This feature may be due to slowly decaying mechanical oscillations in one of the booms.

A slight change in direction of propagation with altitude can be noted, best at downleg where the direction seems to change approximately $20^{\circ}$ in going from 96 to $104 \mathrm{~km}$ altitude. This direction can be due to an influence of shear in a neutral wind, but it should be noted that the measurements of the DC electric fields on board the rocket also show some variations regarding the direction of these fields with altitude (Rinnert, 1992). The uncertainty is too large to allow a quantitative analysis of this variation of direction. It was found that the direction of propagation was essentially horizontal and in the direction of the $\boldsymbol{E} \times \boldsymbol{B}$ direction. A more accurate estimate of the direction of wave propagation can be obtained from an analysis of the variation of the 


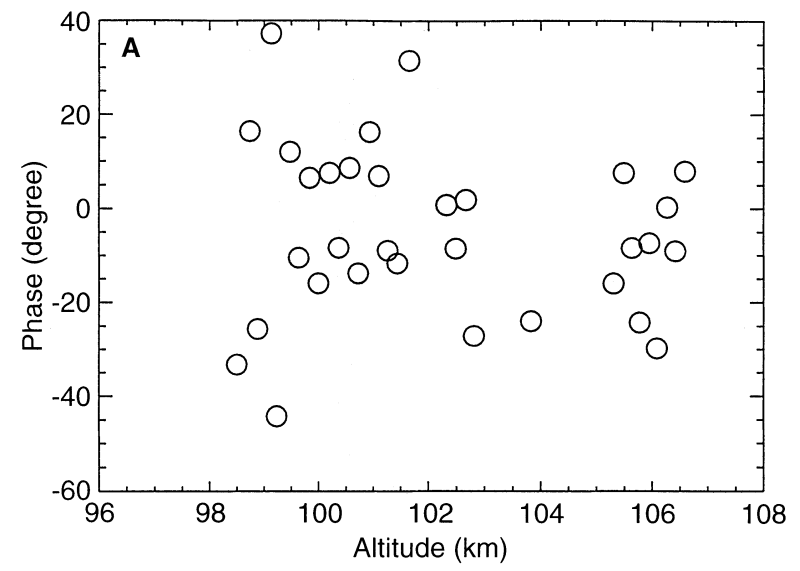

Fig. 11A, B. Relative variation in the spin/coning phase of the rocket at times where the $U_{1}$ and $U_{2}$ probe pairs are aligned to give maximum time-delay for the cross-correlation. The phases are shown

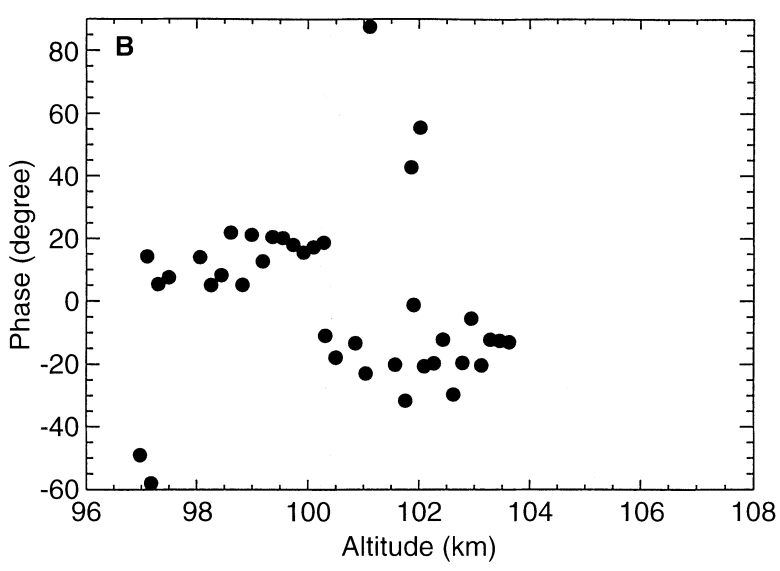

as deviations from the average value determined by ignoring points with large excursion. A refers to upleg, $\mathbf{B}$ to downleg conditions

a time, in an interval of approximately 2 s. Taking the average of the cross-correlation coefficient over several spin periods, we obtain results shown in Fig. 12a, demonstrating that the cross-correlation coefficient has its maximum at the lowest frequencies, and then steadily decreases until a level of $0.2-0.3$ is reached. The same problem can be analyzed by the cross-power spectrum which is here normalized by the square-root of the product of the spectral powers associated with the individual probes, i.e, $S_{12}(\omega) / \sqrt{S_{1}(\omega) S_{2}(\omega)}$. Results are shown in Fig. 12b. For this analysis we used the same data as for the results in Fig. 12a, again with data windows of $100 \mathrm{~ms}$ duration.

Intuitively, we expect that the coherence length of a wave component is proportional to its wavelength, at least in a first approximation. Indeed we find that the longest wavelengths (i.e. smallest frequencies) have the largest coherence (Fig. 12a). The same features are recovered in the results from the cross-power spectra, apart from the lowest frequencies on the upleg part of the flight. Some details in the coherence at large frequencies in Fig. 12b seem to be approximately identical for up- and downleg conditions. As the wave spectrum is unlikely to be so stable over such a long region, we expect that these details are due either to the detecting systems, or to some features generated by the rocket itself. These details in the spectrum appear, however, at frequencies so large that the fluctuation intensities as measured by the power spectra (see Fig. 13) are small, and the effect is therefore of little interest for the present study. Physically, the fading coherence means that the wave components in the highfrequency bands change their statistical properties completely during the time it takes the fluctuation to pass from one probe pair to another.

We notice that the peaks in the power spectra are found for frequencies in the range of $50-100 \mathrm{~Hz}$. With the observed velocities of $200-250 \mathrm{~m} / \mathrm{s}$ this corresponds to wavelengths of $2.5-5 \mathrm{~m}$. Similarly, observed correlation times of $\sim 75 \mathrm{~ms}$ for the signals $U_{5}$ and $U_{6}$ and a rocket speed of $10^{3} \mathrm{~m} / \mathrm{s}$, translates to a characteristic 


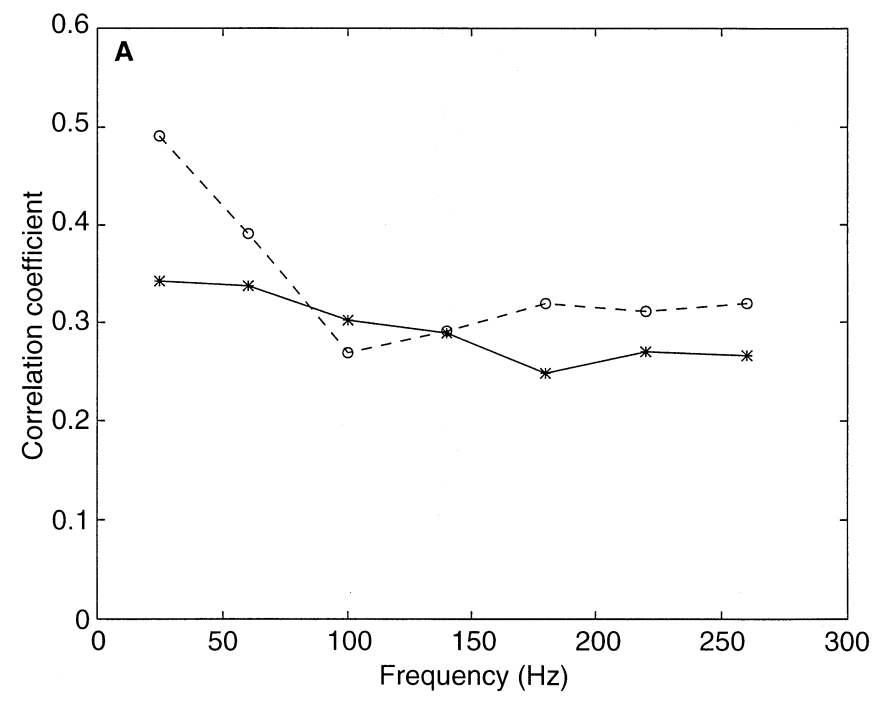

Fig. 12A, B. Cross-coherence of the waves as a function of frequency. The results in $\mathbf{A}$ are obtained by band-pass filtering the data, as when the results in Fig. 10 were obtained. In $\mathbf{B}$ we show the results obtained

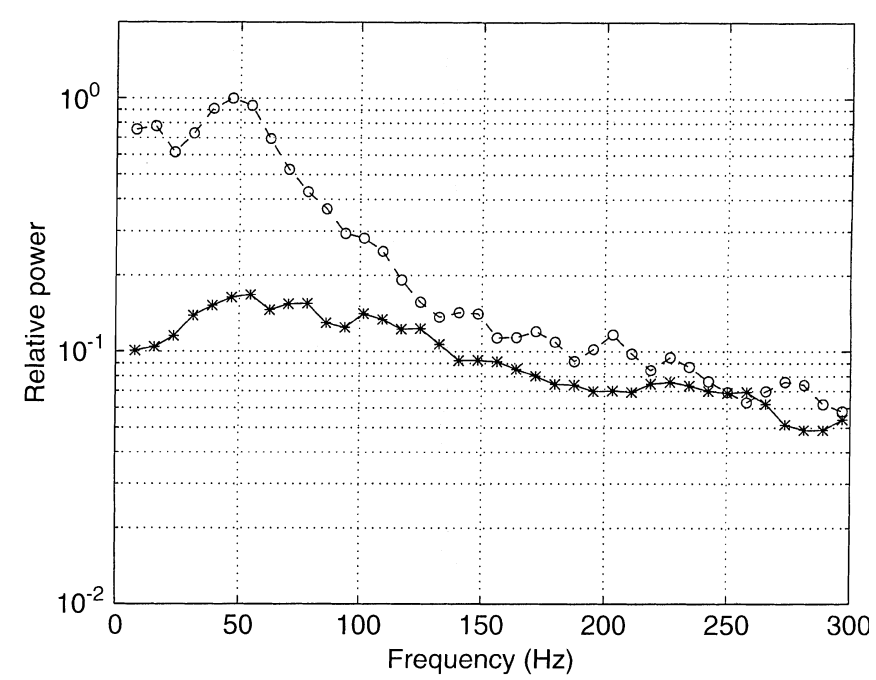

Fig. 13. Power-spectra associated with the electric field signals shown on relative scale, see also Fig. 5. Solid line with asterisks refers to upleg conditions, dashed line with open circles is for downleg. Some fine structure is lost, as these spectra represent averages over approximately $2 \mathrm{~s}$, i.e., several spin periods. The data refer to an altitude interval of approximately $2 \mathrm{~km}$, centered around $102 \mathrm{~km}$ altitude

scale length of $\sim 75 \mathrm{~m}$ for the fluctuations in the direction along the rocket axis. It is consistent with these observations to consider the dominant perturbations in plasma density as "pancake-like", narrow in the direction of their propagation and wide along the direction of the rocket trajectory. Fluctuation spectra of electric fields in the auroral electrojet were reported also by Pfaff et al. (1984). They observed peaks in the spectra around $100 \mathrm{~Hz}$, typically, but their estimate of the propagation velocity is approximately a factor 3 larger than that determined in the present study.

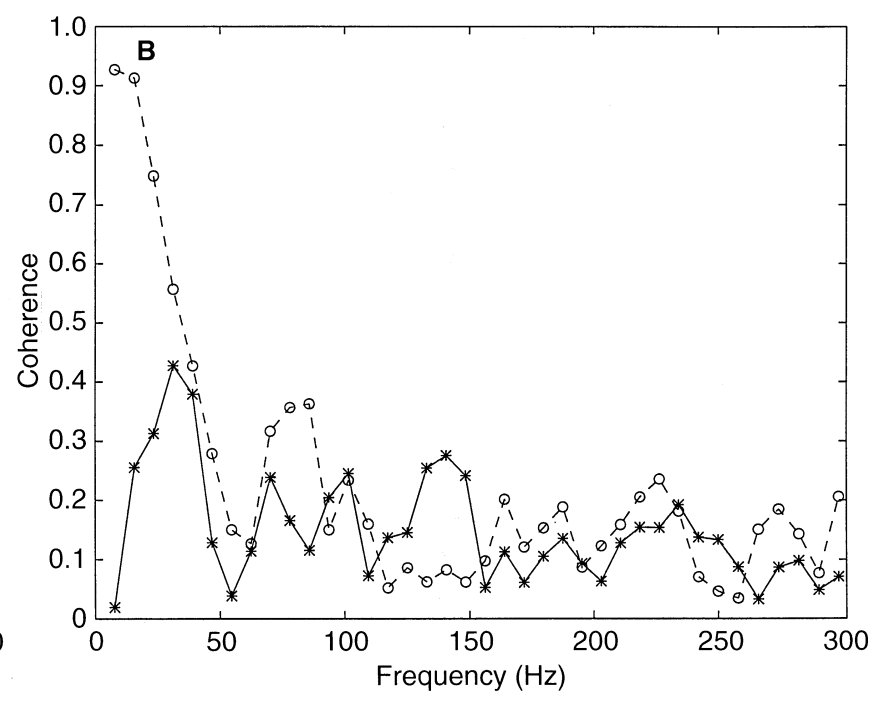

by analyzing the same data in terms of the cross-power spectrum normalized by the square root of the product of the corresponding auto-power spectra

\section{Conclusions}

In the present study we analyzed data from the ROSE F4 rocket. The fluctuations propagate horizontally in a well-defined direction almost perpendicular to the rocket axis. Characteristic propagation speeds of the fluctuations were obtained. It was demonstrated that the fluctuations are dispersive waves with the longer wavelengths having the smaller phase velocity, the smaller wavelength fluctuations propagating almost at the ion sound speed. Within the experimental uncertainty, we found that all frequency components propagated in the same direction. These results were first obtained by band-pass filtering the data and they were confirmed by the cross-phase spectrum obtained for the same data. It was also demonstrated that the coherence of the waves as detected by two probes was approximately proportional to their wavelength, a result which could be expected intuitively.

The main new result presented here is the observation of wave dispersion, speaking against an interpretation in terms of simple acoustic-like waves. Also the sign of dispersion, phase velocities increasing with frequency, is unexpected for acoustic waves. The average phase velocity is significantly below what we would expect from an interpretation in terms of the two-stream instability. Phase velocities below the ion sound speed have been observed before by investigations of rocket data (e.g. Bahnsen et al., 1978; Pécseli et al., 1993). It is unlikely that Doppler shifts induced by neutral winds can explain these low phase velocities. It is also difficult to see how the observed dispersion can be attributed to such effects, even when a neutral wind-shear is allowed for. Local gradients in plasma density are then the most likely candidate for the observed deviations from acoustic-like wave propagation. However, we see one problem in this interpretation. In order for a gradient in 
the direction perpendicular to the magnetic field to have an appreciable influence on the wave characteristics, in particular its dispersion, Eqs. (3) and (4) indicate that a necessary requirement is $k \leq k_{0}$. With the parameters summarized in Table 1 , we find $\varphi=v_{e} v_{i} / \Omega_{e} \Omega_{i} \approx 5 \cdot 10^{-4}$ with $k_{\|} \approx 0$ and also $k_{0} L_{n} \approx 3-4$. Since the dominant wavelength in the observed spectra seem to be comparable to or less than $10 \mathrm{~m}$, a characteristic wave number is $k \sim 1 \mathrm{~m}^{-1}$ and consequently a density gradient should have a local scale-length of the order of $5 \mathrm{~m}$ or less to be of significance for the observed waves, at least according to Eqs. (3) and (4). The relevant density variations should have the character of a relatively fine and almost vertical structure in the relevant part of the $\mathrm{E}$ region (see Fig. 3). The observed velocities and dispersion are similar for up- and downleg conditions, and this interpretation thus assumes such fine structures to be prevalent throughout the $25-\mathrm{km}$ horizontal separation of the up- and downleg parts of the flight. The density measurements on the rocket might indicate small-scale DC density variations (Rose et al., 1992), but since the angle between the rocket trajectory and the magnetic field is small (see Fig. 3), it is difficult to deduce an estimate for density gradients in the $\boldsymbol{B}$-perpendicular direction. Cylindrical DC density variations, i.e, "cigarshaped" irregularities aligned with the magnetic field with a distribution of angles between density gradient and the $\boldsymbol{E} \times \boldsymbol{B}$ direction, are expected to give a significant scatter in the direction of wave-propagation, in disagreement with our observations.

Referring to the study of Sudan (1983), it has been argued by, e.g., Primdahl and Bahnsen (1985) that by introducing an enhanced or anomalous electron collision frequency caused by the fluctuations, the analytical dispersion relation can be made to agree well with observed wave-propagation characteristics, at least those concerning the two-stream instability. We find it interesting that if the electron-neutral collision frequency $v_{e}$ is replaced by a much larger effective value $v_{e f f}$, the observations concerning wave propagation, the wave dispersion in particular, can be made to agree with Eq. (3) at least formally also for large-scale realistic DCdensity variations. In this case we require $\varphi>1$, i.e., $v_{e f f} \gg v_{e}$.

It would be interesting to re-analyze existing data showing exceptionally low velocities of propagation by methods used in the present study, to see whether evidence for non-trivial wave dispersion can be found there too. It should again be emphasized that the plasma perturbations which give the strongest contributions to a correlation analysis are those with largest amplitude in the relevant frequency range, in contrast to those observed by radar scattering measurements where a component of the wave spectrum is selected by a wavenumber matching condition.

Acknowledgements. The sounding rocket project "ROcket and $S$ catter Experiment" organized by G. Rose has been performed in the framework of the German national sounding rocket programme with international participation. It was primarily funded by the Bundesministerium für Forschung and Technologie
(BMFT), and was managed by the Deutsche Gesellschaft für Luftund Raumfahrt (DLR). The present study was supported in part by the Norwegian Research Council (NFR).

Topical Editor D. Alcaydé thanks R. F. Pfaff Jr. and J. D. Sahr for their help in evaluating this paper.

\section{References}

Bahnsen, A., E. Ungstrup, C.-G. Fälthammer, U. Fahleson, J. K. Olesen, F. Primdahl, F. Spangslev, and A. Pedersen, Electrostatic waves observed in an unstable polar cap ionosphere, J. Geophys. Res., 83, 5191, 1978.

Buneman, O., Excitation of field-aligned sound waves by electron streams, Phys. Rev. Lett., 10, 285, 1963.

Farley, D. T., Two-stream plasma instability as a source of irregularities in the ionosphere, Phys. Rev. Lett., 10, 279, 1963.

Friker, A., and F.-J. Lübken, Neutral air density and temperature measurements by the TOTAL instrument onboard the ROSE payloads, J. Atmos. Terr. Phys., 54, 693, 1992.

Iranpour, K., H. L. Pécseli, J. Trulsen, A. Bahnsen, F. Primdahl, G. Rose, and K. Rinnert, Low-frequency electrostatic waves in the ionospheric E-region. Proceedings from the Mini-workshop on Wave Phenomena in Solar-Terrestrial Plasmas, Institute for Theoretical Astrophysics, The University of Oslo, Norway, 14-16 June, 1993, Eds. P. Maltby and H. L. Pécseli, Institute of Astrophysics Report, pp. 181-188, 1993.

John, P. J., and Y. A. Saxena, Observations of the FarleyBuneman instability in a laboratory plasma, Geophys. Res. Lett, 2, 251, 1975.

Kohl, H, E. Nielsen, K. Rinnert, and K. Schlegel, EISCAT results during the ROSE campaign and comparison with STARE measurements, J. Atmos. Terr. Phys., 54, 733, 1992.

Kelley, M. C., and F. S. Mozer, Electric field and plasma density oscillations due to the high-frequency Hall current two-stream instability in the Auroral E region, J. Geophys. Res., 78, 2214, 1973.

Kudeki, E., D. T. Farley, and B. Fejer, Long wavelength irregularities in the equatorial electrojet, Geophys. Res. Lett., 9, 684, 1982.

LaBelle, J., P. M. Kintner, and M. C. Kelley, Interferometric phase velocity measurements in the Auroral electrojet, Planet. Space Sci, 34, 1285, 1986.

Mikkelsen, T., and H. L. Pécseli, Strong turbulence in partially ionized plasmas, Phys. Lett. A, 77, 159, 1980.

Pécseli, H. L., and J. Trulsen, On the interpretation of experimental methods for investigating nonlinear wave phenomena, Plasma Phys. Contr. Fusion, 35, 1701, 1993.

Pécseli, H. L., T. Mikkelsen, and S. E. Larsen, Drift wave turbulence in low- $\beta$ plasmas, Plasma Phys, 25, 1173, 1983.

Pécseli, H. L., F. Primdahl, and A. Bahnsen, Low-frequency electrostatic turbulence in the polar cap E-region, J. Geophys. Res., 94, 5337, 1989.

Pécseli, H. L., J. Trulsen, A. Bahnsen, and F. Primdahl, Propagation and nonlinear interaction of low-frequency electrostatic waves in the polar cap E region, J. Geophys. Res., 98, 1603, 1993.

Pfaff, R. F., M. C. Kelley, B. G. Fejer, E. Kudeki, C. W. Carlson, A. Pedersen, and B. Hausler, Electric field and plasma density measurements in the Auroral electrojet, J. Geophys. Res., 89, 236, 1984.

Pfaff, R. F., J. Sahr, J. F. Providakes, W. E. Swartz, D. T. Farley, P. M. Kintner, I. Häggström, A. Hedberg, H. Opgenoorth, G. Holmgren, A. McNamara, D. Wallis, B. Whalen, A. Yau, S. Watanabe, F. Creutzenberg, P. Williams, E. Nielsen, K. Schlegel and T. R. Robinson, The E-region rocket/radar instability study (ERRIS): scientific objectives and campaign overview, J. Atmos. Terr. Phys., 54, 779, 1992. 
Primdahl, F., Polar ionospheric E-region plasma wave stabilization and electron heating by wave-induced enhancements of the electron collision frequency, Phys. Scr. 33, 187, 1986.

Primdahl, F., and A. Bahnsen, Auroral E-region diagnosis by means of nonlinearly stabilized plasma waves, Ann. Geophysicae, 3, 57, 1985.

Rees, D., and T. J. Fuller-Rowell, Geomagnetic response of the polar thermosphere and ionosphere, in Electromagnetic Coupling in the Polar Clefts and Caps, Eds. P. E. Sandholdt and A. Egeland, Kluwer, Dordrecht, p. 355, 1988.

Rinnert, K., Plasma waves observed in the Auroral E-region ROSE campaign, J. Atmos. Terr. Phys., 54, 683, 1992.

Rogister, A., Nonlinear theory of cross-field instability with application to the equatorial electrojet, J. Geophys. Res, 77, 2975, 1972.
Rohde, V., A. Piel, H. Thiemann, and K. I. Oyama, In situ diagnostics of ionospheric plasma with the resonance cone technique, J. Geophys. Res, 98, 19163, 1993.

Rose, G., K. Schlegel, K. Rinnert, H. Kohl, E. Nielsen, G. Dehmel, A. Friker, F.-J. Lübken, H. Lühr, E. Neske, and A. Steinweg, The ROSE project. Scientific objectives and discussion of first results, J. Atmos. Terr. Phys., 54, 657, 1992.

Sudan, R. N., Unified theory of type-I and type-II irregularities in the equatorial electrojet, J. Geophys. Res., 88, 4853, 1983.

Sudan, R. N., J. Akinrimisi, and D. T. Farley, Generation of smallscale irregularities in the equatorial electrojet, J. Geophys. Res, 78, 240, 1973. 